\title{
NIMO-CKD-UK: a real-world, observational study of iron isomaltoside in patients with iron deficiency anaemia and chronic kidney disease
}

Philip A. Kalra ${ }^{1 *}$, Sunil Bhandari ${ }^{2}$, Michael Spyridon ${ }^{3}$, Rachel Davison ${ }^{4}$, Sarah Lawman ${ }^{5}$, Ashraf Mikhail ${ }^{6}$, David Reaich ${ }^{7}$, Nick Pritchard ${ }^{8}$, Kieran McCafferty ${ }^{9}$ and Jason Moore ${ }^{10}$

\begin{abstract}
Background: Intravenous iron is often used to treat iron deficiency anaemia in non-dialysis chronic kidney disease (ND-CKD), but the optimal dosing regimen remains unclear. We evaluated the impact of high-versus low-dose intravenous iron isomaltoside on the probability of retreatment with intravenous iron in iron-deficient ND-CKD patients.

Methods: This real-world, prospective, observational study collected data from 256 ND-CKD patients treated for anaemia in the UK. Following an initial course of iron isomaltoside, patients were followed for $\geq 12$ months. Iron dose and the need for retreatment were determined at the investigators' discretion. The primary study outcome was the need for retreatment at 52 weeks compared between patients who received $>1000 \mathrm{mg}$ of iron during Course 1 and those who received $\leq 1000 \mathrm{mg}$. Safety was evaluated through adverse drug reactions.

Results: The probability of retreatment at Week 52 was significantly lower in the $>1000 \mathrm{mg}$ iron group $(n=58)$ versus the $\leq 1000 \mathrm{mg}$ group ( $n=198)$; hazard ratio (95\% confidence interval [CI]): $0.46(0.20,0.91) ; p=0.012$. Mean $(95 \% \mathrm{Cl})$ haemoglobin increased by $6.58(4.94,8.21) \mathrm{g} / \mathrm{L}$ in the $\leq 1000 \mathrm{mg}$ group and by $10.59(7.52,13.66) \mathrm{g} / \mathrm{L}$ in the $>1000 \mathrm{mg}$ group $(p=0.024)$. Changes in other blood and iron parameters were not significantly different between the two groups. Administering $>1000 \mathrm{mg}$ of iron isomaltoside saved 8.6 appointments per 100 patients compared to $\leq 1000 \mathrm{mg}$. No serious adverse drug reactions were reported. Of the patients who received $\leq 1000 \mathrm{mg}$ of iron in this study, $82.3 \%$ were eligible for a dose $>1000 \mathrm{mg}$.

Conclusions: The $>1000 \mathrm{mg}$ iron isomaltoside regimen reduced the probability of retreatment, achieved a greater haemoglobin response irrespective of erythropoiesis-stimulating agent treatment, and reduced the total number of appointments required, compared to the $\leq 1000 \mathrm{mg}$ regimen. Many of the patients who received $\leq 1000 \mathrm{mg}$ of iron were eligible for $>1000 \mathrm{mg}$, indicating that there was considerable underdosing in this study.
\end{abstract}

Trial registration: ClinicalTrials.gov NCT02546154, 10 September 2015.

Keywords: Anaemia, Chronic kidney disease, Ferric derisomaltose, Intravenous iron, Iron deficiency, Iron isomaltoside 1000, Non-dialysis dependent, Observational, Real-world

\footnotetext{
* Correspondence: philip.kalra@srft.nhs.uk

'Salford Royal NHS Foundation Trust, Salford, UK

Full list of author information is available at the end of the article
}

(c) The Author(s). 2020 Open Access This article is licensed under a Creative Commons Attribution 4.0 International License, which permits use, sharing, adaptation, distribution and reproduction in any medium or format, as long as you give appropriate credit to the original author(s) and the source, provide a link to the Creative Commons licence, and indicate if changes were made. The images or other third party material in this article are included in the article's Creative Commons licence, unless indicated otherwise in a credit line to the material. If material is not included in the article's Creative Commons licence and your intended use is not permitted by statutory regulation or exceeds the permitted use, you will need to obtain permission directly from the copyright holder. To view a copy of this licence, visit http://creativecommons.org/licenses/by/4.0/ The Creative Commons Public Domain Dedication waiver (http://creativecommons.org/publicdomain/zero/1.0/) applies to the data made available in this article, unless otherwise stated in a credit line to the data. 


\section{Background}

Iron deficiency anaemia (IDA) is common in chronic kidney disease (CKD) [1], and studies have shown that the prevalence of anaemia increases with declining kidney function [2, 3]. Untreated anaemia can lead to a reduced quality of life [4], disease progression [5, 6], and adverse clinical outcomes [7].

Intravenous (IV) iron can be used to treat anaemia in non-dialysis CKD (ND-CKD) patients, as oral iron is not tolerated by some individuals, and is less efficacious in the more advanced stages of CKD (CKD stages 4 and 5) [7-9]. However, the optimal IV iron regimen in ND-CKD remains unclear. Guidance for anaemia management from the UK National Institute for Health and Care Excellence (NICE) recommends offering high-dose, low-frequency IV iron therapy to ND-CKD patients, stating that a minimum of $500-1000 \mathrm{mg}$ of iron should achieve iron repletion in most patients [7]. On the other hand, evidence from several large randomised controlled trials (RCTs) indicates that the iron deficit in patients with IDA is often $>1000 \mathrm{mg}[10,11]$.

Data on the effectiveness of different IV iron regimens in ND-CKD patients are limited. However, in the dialysis population, the PIVOTAL study - an RCT of 2141 UK haemodialysis patients - showed that higher doses of IV iron were more effective than lower doses on the primary composite endpoint of non-fatal myocardial infarction, non-fatal stroke, hospitalisation for heart failure, or death from any cause [12]. In the non-dialysis population, the REPAIR-IDA study - an RCT in 2584 NDCKD patients - showed that higher doses of IV iron (1500 mg ferric carboxymaltose) resulted in greater increases in haemoglobin $(\mathrm{Hb})$ and iron parameters compared to lower doses (1000 mg iron sucrose), with similar safety observations reported between the two groups [13]. Taking this one step further, the FINDCKD study - an RCT in 626 ND-CKD patients - concluded that targeting high ferritin levels by using higher doses of IV iron, delayed and/or reduced the need for further anaemia management [14]. However, few studies have evaluated the real-world impact of higher versus lower IV iron dosing regimens in the management of anaemia in ND-CKD patients.

Iron isomaltoside 1000/ferric derisomaltose (IIM) (produced by Pharmacosmos A/S, Denmark; marketed as Monofer ${ }^{\odot} /$ Monoferric $^{\circ}$ ) is an IV iron preparation delivered in single, high doses of up to $20 \mathrm{mg} / \mathrm{kg}$ body weight [15]. Data from clinical trials have confirmed the good efficacy and safety profile of IIM in patients with CKD [8, 16-19]. In addition, observational studies in CKD conducted in Germany [20], Scandinavia [21], and the UK [22], have demonstrated that IIM is well tolerated and effective in real-world settings.

The purpose of the 'Non-Interventional Monofer' (NIMO)-CKD-UK' study was to investigate the impact of high- versus low-dose IIM on the probability of retreatment with IV iron in ND-CKD patients, across different UK hospitals. This pragmatic, real-world study also evaluated the real-world effectiveness of IIM in UK clinical practice.

\section{Methods}

\section{Study design and population}

Participants were recruited from 11 UK hospitals into a prospective, observational study, conducted between January 2016 and December 2018 (ClinicalTrials.gov registry: NCT02546154). The study population included ND-CKD patients (aged $\geq 18$ years) who were diagnosed with IDA according to local hospital guidelines; the study criteria required that the IDA be considered a consequence of CKD. Generally, hospitals in the UK adhere to NICE or Kidney Disease Improving Global Outcomes (KDIGO) clinical guidelines for the management of anaemia in CKD, which recommend using a combination of serum ferritin and transferrin saturation (TSAT) measurements to diagnose iron deficiency [7, 23].

After an initial course of IIM, patients were followed for $\geq 52$ weeks to capture retreatment with IV iron according to local hospital practice. The decision to retreat with IV iron and the patient-specific dosing regimen were based on $\mathrm{Hb}$ and/or iron parameters at the time of retreatment, and were at the discretion of each study investigator, in accordance with local clinical practice. The study terminated once the 52-week observational period had been completed and the last blood sample had been collected from the last patient treated with IIM. Patients who reached the end of the 52-week follow-up period having received at least two courses of IV iron completed the study. The remaining patients were followed beyond Week 52 until a second course of IV iron occurred or until study termination. Consequently, the observation period varied between patients and, for some patients, was longer than 52 weeks. Patients were discontinued if they became haemodialysis-dependent, or if they received an alternative IV iron therapy during the study course.

\section{Data collection and outcome measures}

Data for IV iron doses and for blood and iron parameters were collected from patient medical records. Blood samples were taken before IV iron administration and 4-6 weeks after treatment, according to local clinical practice. Patient data were used to estimate iron need using the simplified table and the Ganzoni formula (iron need $=$ patient weight $\mathrm{x}$ [target $\mathrm{Hb}-$ current $\mathrm{Hb}] \times 0.24+500)$ [24], to allow for a comparison between the estimated iron need and the dose of iron that the patient received (both methods are outlined in the product label for IIM [15]). Quality of life 
(fatigue symptoms) was assessed before IV iron administration and approximately 4 weeks after treatment, using the 13-item Functional Assessment of Chronic Illness Therapy-Fatigue (FACIT-Fatigue) scale (scored from 0 to 52; higher score indicates better quality of life) [25].

The primary study outcome was the probability of retreatment with IV iron, according to local hospital practice, at Week 52. Secondary outcomes included the probability of retreatment at 6-monthly intervals, the mean change from baseline in blood/iron parameters and in the FACIT-Fatigue questionnaire, and the proportion of patients with an $\mathrm{Hb}$ level of $\geq 110 \mathrm{~g} / \mathrm{L}$ (the threshold for anaemia investigation and management in CKD patients, according to the NICE guideline) [7]. Exploratory outcomes included a breakdown of the $\mathrm{Hb}$ data according to treatment with erythropoiesisstimulating agents (ESAs) at baseline, and the number of appointments required in each dose group. All adverse drug reactions (ADRs) were reported to the Sponsor's pharmacovigilance department, and in accordance with the national reporting systems.

\section{Statistical methods}

Data analyses were conducted for all patients who received IIM during the initial treatment course. Analyses were conducted based on comparisons between two dose groups, according to the amount of iron received during the first treatment course (Course 1) $-\leq 1000 \mathrm{mg}$ or $>1000 \mathrm{mg}$. Selected baseline demographics and clinical characteristics were compared between the groups using a two-sided, two sample t-test assuming unequal variability (weight, kidney function, $\mathrm{Hb}$, ferritin, TSAT, platelets, ESA dose, and FACIT-Fatigue Total score) or a two-sided Fisher's Exact Test (gender). The primary outcome - probability of retreatment with IV iron at Week 52 - was analysed using a Cox proportional hazards model, with dose group as a factor, and baseline $\mathrm{Hb}$ as a covariate. Patients who received only one IV iron course were censored at study end. Data on the primary outcome from patients who discontinued due to receiving an alternative IV iron therapy were included in the primary outcome analysis. The two-sided 95\% confidence interval $(\mathrm{CI})$ for the difference in probability of retreatment between the dose groups was calculated and the corresponding $p$-value was derived (based on the pooled standard error estimated from the Cox proportional hazards model). Mean change estimates for blood/iron parameters and FACIT-Fatigue questionnaire before and after treatment were obtained from an analysis of covariance (ANCOVA) model with dose group as a factor, and pre-treatment value as a covariate. Comparisons between groups from the ANCOVA model were twosided. For the proportion of patients with an $\mathrm{Hb}$ level $\geq 110 \mathrm{~g} / \mathrm{L}$, odds ratios were calculated, and two-sided $p$ values were obtained from a Fisher's Exact Test. The significance cut-off for all analyses was $p<0.05$. No formal statistical analyses were performed on the exploratory outcomes. Missing data were not accounted for in the analyses.

Safety was evaluated through the reporting of ADRs.

\section{Results}

\section{Patient population}

A total of 256 patients were recruited into the study; $77.3 \%$ of patients received $\leq 1000 \mathrm{mg}$ in Course 1 (Fig. 1).

Patient demographics and baseline clinical characteristics are presented in Table 1.

The proportion of male patients was significantly higher in the $>1000 \mathrm{mg}$ dose group than $\leq 1000 \mathrm{mg}$ dose group $(p=0.025)$. Patients in the $>1000 \mathrm{mg}$ dose group were significantly heavier than patients in the $\leq 1000 \mathrm{mg}$ dose group $(p<0.001)$. Baseline kidney function, $\mathrm{Hb}$, TSAT and platelets were similar between the two dose groups. Additional blood and iron parameters were also comparable between the groups (Additional file 1, Table S1; no formal statistical analyses were performed). Ferritin was significantly higher in the $\leq 1000 \mathrm{mg}$ dose group $(p=0.022)$. The proportion of patients not receiving ESAs was similar between the two groups ( $\leq 1000 \mathrm{mg}$ : $78.3 \%$; $>1000 \mathrm{mg}$ : $74.1 \%$ ); of those patients who were receiving ESA the mean cumulative monthly dose of ESA was numerically higher in patients receiving $\leq 1000 \mathrm{mg}$ IIM.

\section{Iron dosing and treatment routine}

The patient data collected were used to estimate the iron need in patients using the simplified table or the Ganzoni formula [15, 24]. Based on these calculations, the actual dose of IIM administered during Course 1 in the $\leq 1000 \mathrm{mg}$ group was approximately half of the iron requirement estimated, whereas in the $>1000 \mathrm{mg}$ group, the actual dose received was close to the estimated iron need (Table 2). Of the patients who received $\leq 1000 \mathrm{mg}$, $82.3 \%$ were eligible to receive a dose $>1000 \mathrm{mg}$ based on their baseline $\mathrm{Hb}$ and weight (Table 2).

Within each dose group, the mean dose of IIM was not affected by whether patients were receiving ESA at baseline or not (Additional file 1, Table S2).

\section{Probability of retreatment with IV iron}

Following a first course of IIM, the probability of no retreatment over time, according to local hospital practice, is presented in Fig. 2.

At Week 52, the probability of retreatment with IV iron was significantly lower in the $>1000 \mathrm{mg}$ group than in the $\leq 1000 \mathrm{mg}$ group - hazard ratio (HR): 0.46 (95\% CI: 0.20, $0.91) ; p=0.012$. A statistically significant difference in the probability of retreatment was observed between the two 


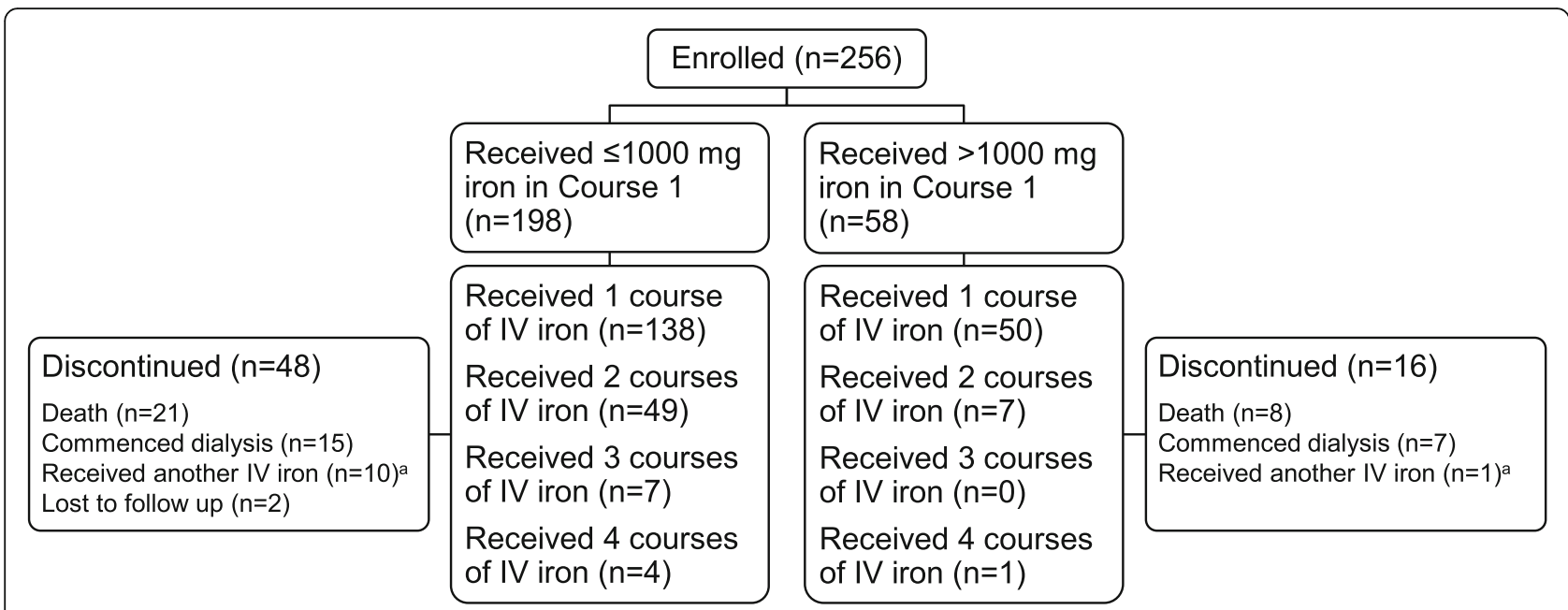

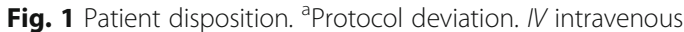

dose groups at all timepoints assessed, up to Week 104 (Additional file 1, Table S3).

\section{Effectiveness}

The change in $\mathrm{Hb}$ from baseline to after Course 1 was significantly greater in the $>1000 \mathrm{mg}$ dose group (least squares [LS] mean: 10.59 [95\% CI: 7.52, 13.66] g/L) versus the $\leq 1000 \mathrm{mg}$ group (LS mean: 6.58 [95\% CI: 4.94, 8.21] g/L) $(p=0.024)$; no statistically significant difference from baseline was observed after Course 2 (Table 3). Data for additional courses are not presented as very few patients received more than two courses. Within each dose group, the changes in $\mathrm{Hb}$ were not affected by ESA treatment at baseline (Additional file 1, Table S4). No significant differences between the two dose groups were observed in the changes before and after treatment for ferritin, TSAT and platelets (Table 3).

After Course 1, the proportion of patients with an $\mathrm{Hb}$ level $\geq 110 \mathrm{~g} / \mathrm{L}$ was greater in the $>1000 \mathrm{mg}$ versus the $\leq 1000 \mathrm{mg}$ group, irrespective of ESA treatment at baseline; the difference was significant in patients not receiving ESA at baseline $(p=0.01)$ (Figs. 3 and 4). A similar trend was observed after Course 2 (Figs. 3 and 4).

Overall, the FACIT-Fatigue score showed a significant improvement from baseline after Course 1, in both dose groups $(p<0.0001)$. The between-group difference in the LS mean change from baseline was not significant (Additional file 1; Table S5).

\section{Use of healthcare resources}

Administering $>1000 \mathrm{mg}$ of IIM during Course 1 saved 8.6 appointments per 100 patients compared with administering $\leq 1000 \mathrm{mg}$, over the duration of the study (Table 4).

During Course 1, all patients in the $\leq 1000 \mathrm{mg}$ group received their prescribed dose in one administration. In the $>1000 \mathrm{mg}$ group, the prescribed dose was given in one administration for $86.2 \%$ of patients and in two administrations for $13.8 \%$ of patients.

\section{Safety}

One non-serious ADR was reported in a total of 336 administrations $(0.3 \%)$ - pruritus and rash were observed in one patient in the lower dose group. No serious ADRs were reported.

A total of 29 (11.3\%) patients died during the study - $21(10.6 \%)$ deaths were reported in the $\leq 1000 \mathrm{mg}$ group and $8(13.8 \%)$ in the $>1000 \mathrm{mg}$ group (Fig. 1). None of the deaths were considered to be related to IIM by the investigators. Overall, there was no difference between the dose groups in the time from the last IV iron infusion to death ( $\leq 1000 \mathrm{mg}$ : 19 weeks; >1000 mg: 21 weeks).

\section{Discussion}

The real-world, observational NIMO-CKD-UK study showed that a higher dose IV iron regimen $(>1000 \mathrm{mg}$ ) decreased the probability of retreatment compared to a lower dose regimen $(\leq 1000 \mathrm{mg})$. This observation supports the findings of the PIVOTAL, FIND-CKD and REPAIR-IDA studies that higher doses of IV iron can be beneficial [12-14].

NIMO-CKD-UK is a unique study comparing the impact of IV iron doses on the probability of retreatment in non-dialysis patients. When calculated using the simplified table or the Ganzoni formula, most patients requiring IV iron would receive an initial dose $>1000 \mathrm{mg}$. Such doses have been shown to reduce the probability of retreatment for anaemia in indications other than CKD. A Scandinavian observational study showed that gastroenterology patients receiving $>1000 \mathrm{mg}$ IIM during an initial course had a $65 \%$ lower probability of retreatment 
Table 1 Patient demographics and baseline clinical characteristics

\begin{tabular}{|c|c|c|c|}
\hline & $\begin{array}{l}\leq 1000 \mathrm{mg} \\
(N=198)\end{array}$ & $\begin{array}{l}>1000 \mathrm{mg} \\
(N=58)\end{array}$ & $p$-value \\
\hline \multicolumn{4}{|l|}{ Demographics } \\
\hline Male, n (\%) & $96(48.5)$ & $38(65.5)$ & 0.025 \\
\hline \multicolumn{4}{|l|}{ Weight, kg } \\
\hline Mean (SD) & $77.1(20.07)$ & $89.7(19.40)$ & $<0.001$ \\
\hline$\geq 50$ kg, n (\%) & $179(90.4)$ & $58(100.0)$ & \\
\hline$\geq 75$ kg, n (\%) & $94(47.5)$ & $47(81.0)$ & \\
\hline \multicolumn{4}{|l|}{ Clinical characteristics } \\
\hline \multicolumn{4}{|l|}{$\mathrm{eGFR}, \mathrm{mL} / \mathrm{min} / 1.73 \mathrm{~m}^{2}$} \\
\hline Mean (SD) & $25.4(32.37)$ & $25.7(11.93)$ & 0.910 \\
\hline$>30, \mathrm{n}(\%)$ & $39(19.7)$ & $13(22.4)$ & \\
\hline$\geq 15$ to $\leq 30, \mathrm{n}(\%)$ & $122(61.6)$ & $38(65.5)$ & \\
\hline$<15, \mathrm{n}(\%)$ & $37(18.7)$ & $7(12.1)$ & \\
\hline $\mathrm{Hb}, \mathrm{g} / \mathrm{L}(\mathrm{n})$ & (198) & $(57)$ & \\
\hline Mean (SD) & $100.6(11.65)$ & $102.5(10.92)$ & 0.263 \\
\hline Ferritin, $\mu \mathrm{g} / \mathrm{L}$ (n) & (157) & $(45)$ & \\
\hline Mean (SD) & $161.9(188.74)$ & $111.9(103.19)$ & 0.022 \\
\hline Median (Q1, Q3) & $106.0(52.0,170.0)$ & $68.0(39.0,137.0)$ & \\
\hline TSAT, \% (n) & (97) & (31) & \\
\hline Mean (SD) & $15.8(6.58)$ & $13.7(6.03)$ & 0.108 \\
\hline Platelets, $\times 10^{9} / \mathrm{L}(\mathrm{n})$ & (143) & $(45)$ & \\
\hline Mean (SD) & $249.9(88.12)$ & $222.1(96.99)$ & 0.091 \\
\hline \multicolumn{4}{|l|}{ Concomitant ESA, IU } \\
\hline Receiving ESA, n (\%) & $43(21.7)$ & $15(25.9)$ & \\
\hline \multicolumn{4}{|l|}{ Cumulative monthly ESA dose $\mathrm{e}^{\mathrm{b}}$} \\
\hline Mean (SD) & 9505 (12907) & $7570(4963)$ & 0.419 \\
\hline Median & 6000 & 7000 & \\
\hline FACIT-Fatigue Total score (n) & (196) & $(58)$ & \\
\hline Mean (SD) & $25.5(13.71)$ & $24.1(13.06)$ & 0.491 \\
\hline
\end{tabular}

eGFR estimated glomerular filtration rate, ESA erythropoiesis-stimulating agent, FACIT Functional Assessment of Chronic Illness Therapy, Hb haemoglobin, IU international unit, $n$ number of patients with data, Q1 lower quartile of the interquartile range, Q3 upper quartile of the interquartile range,

$S D$ standard deviation, TSAT transferrin saturation

${ }^{a}$ ESA were administered either intravenously or subcutaneously. Doses expressed in $\mu g$ were converted to IU (dose in IU/L $=$ dose in $\left.\mu g / L \times 200\right)[12,26]$

${ }^{\mathrm{b}}$ Recorded for the 4 weeks prior to study entry

Table 2 Dose of IIM administered during Course 1 - actual dose versus the estimated iron need

\begin{tabular}{|c|c|c|c|c|}
\hline & \multicolumn{2}{|c|}{$\begin{array}{c}\leq 1000 \mathrm{mg} \\
(N=198)\end{array}$} & \multicolumn{2}{|c|}{$\begin{array}{c}>1000 \mathrm{mg} \\
(N=58)\end{array}$} \\
\hline Actual dose received, $\mathrm{mg}$ & \multicolumn{2}{|c|}{$814.4(215.53)$} & \multicolumn{2}{|c|}{$1537.9(185.27)$} \\
\hline \multirow[t]{2}{*}{ Estimated iron need, mg } & Simplified & Ganzoni $^{\mathrm{a}}$ & Simplified & Ganzoni $^{\mathrm{a}}$ \\
\hline & $1539.1(341.23)^{b}$ & $1410.1(305.71)^{c}$ & $1649.1(249.37)^{d}$ & $1521.4(318.41)^{d}$ \\
\hline Difference between actual and estimated dose, mg & $-698.3(387.95)^{b}$ & $-581.7(335.71)^{c}$ & $-108.8(292.94)^{d}$ & $19.0(341.02)^{d}$ \\
\hline Patients eligible for > $1000 \mathrm{mg}$ of iron, $\mathrm{n}(\%)^{\mathrm{e}}$ & \multicolumn{2}{|c|}{$163(82.3)$} & \multicolumn{2}{|c|}{$56(96.6)$} \\
\hline
\end{tabular}

Data presented are mean (SD)

$n$ number of patients with data, $S D$ standard deviation

${ }^{a} \mathrm{~A}$ target $\mathrm{Hb}$ of $150 \mathrm{~g} / \mathrm{L}$ was entered in the Ganzoni formula; ${ }^{b} n=179 ;{ }^{c} n=188 ;{ }^{d} n=57$; eligibility for $>1000$ mg was determined using the simplified dosing table, based on $\mathrm{Hb}$ and weight at baseline 


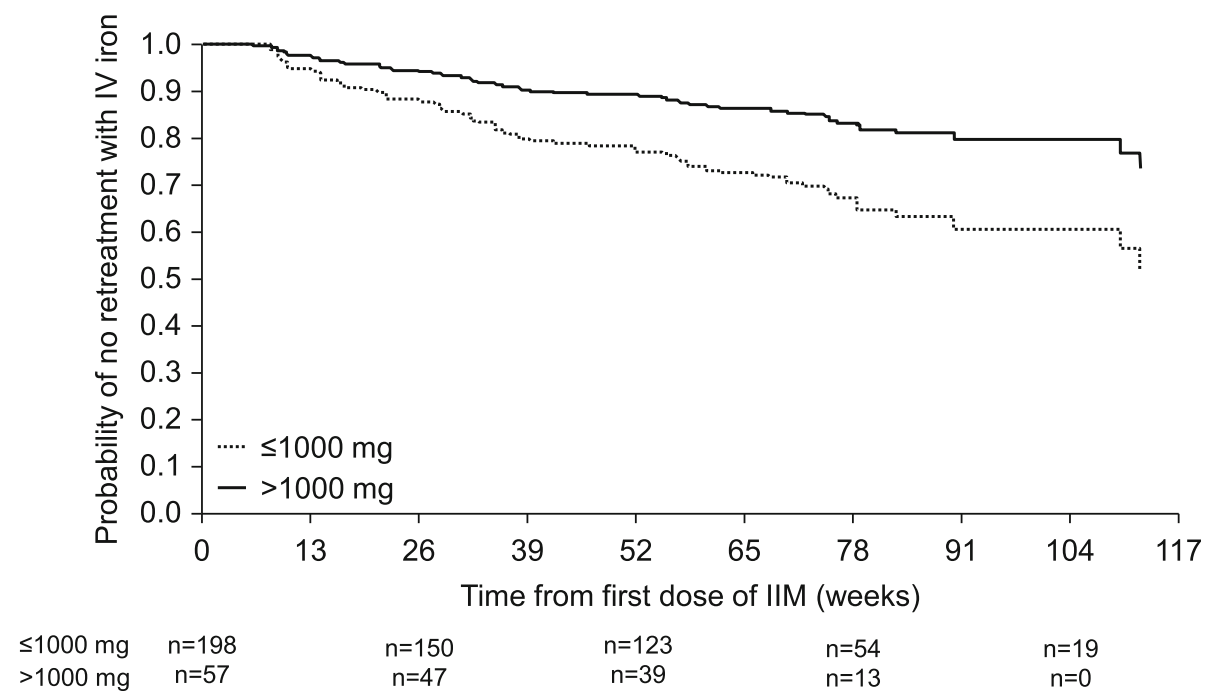

Fig. 2 Probability of no retreatment with IV iron, by IIM dose administered during Course 1. IIM iron isomaltoside, $I V$ intravenous, $n$ number of patients included in the analysis

at Week 52 versus those receiving $1000 \mathrm{mg}$ [27]. Reducing the need for retreatment with IV iron can have a positive impact on the use of healthcare resources and for patients. Indeed, the findings of the NIMO-CKD-UK study suggest fewer hospital appointments with the use of higher versus lower doses of IIM, which could translate into economic savings and alleviate pressures on healthcare systems that often operate near capacity. Current evidence from health economic analyses suggests that IIM can provide cost-savings to the healthcare system through lower resource usage (lower number of infusions per patient and a lower proportion of patients requiring multiple infusions) compared with other treatments [28-31]. Fewer appointments reduce the burden on patients, who may need to travel long distances to attend appointments. In addition, fewer hospital visits could have clear benefits during the COVID-19 pandemic, where social distancing is necessary and reducing pressure on hospital resources is crucial $[32,33]$.

Table 3 Mean change from baseline in blood and iron parameters before and after IIM treatment

\begin{tabular}{|c|c|c|c|c|}
\hline Parameter, by timepoint & $\begin{array}{l}\leq 1000 \mathrm{mg} \\
(N=198)\end{array}$ & $\begin{array}{l}>1000 \mathrm{mg} \\
(N=58)\end{array}$ & $\begin{array}{l}\text { Difference } \\
(95 \% \mathrm{Cl})\end{array}$ & $p$-value \\
\hline \multicolumn{5}{|l|}{$\mathrm{Hb}, \mathrm{g} / \mathrm{L}$} \\
\hline After Course $1(\leq 1000 \mathrm{mg}, n=194 ;>1000 \mathrm{mg}, n=56)$ & $6.58(4.94,8.21)$ & $10.59(7.52,13.66)$ & $4.02(0.54,7.50)$ & 0.024 \\
\hline Before Course $2(\leq 1000 \mathrm{mg}, \mathrm{n}=56 ;>1000 \mathrm{mg}, n=10)$ & $-0.26(-3.61,3.08)$ & $4.96(-2.96,12.88)$ & $5.22(-3.40,13.84)$ & 0.231 \\
\hline After Course $2(\leq 1000 \mathrm{mg}, n=55 ;>1000 \mathrm{mg}, n=7)$ & $8.39(4.63,12.16)$ & $7.79(-2.85,18.43)$ & $-0.60(-11.93,10.73)$ & 0.916 \\
\hline \multicolumn{5}{|l|}{ Ferritin, $\mu \mathrm{g} / \mathrm{L}$} \\
\hline After Course $1(\leq 1000 \mathrm{mg}, n=157 ;>1000 \mathrm{mg}, n=43)$ & $277.98(238.50,317.46)$ & $306.09(227.42,384.75)$ & $28.10(-60.32,116.53)$ & 0.531 \\
\hline Before Course $2(\leq 1000 \mathrm{mg}, n=45 ;>1000 \mathrm{mg}, n=7)$ & $91.50(55.12,127.88)$ & $120.25(4.72,235.77)$ & $28.74(-93.71,151.19)$ & 0.638 \\
\hline After Course $2(\leq 1000 \mathrm{mg}, n=42 ;>1000 \mathrm{mg}, n=6)$ & $458.23(317.77,598.70)$ & $424.37(-177.2,1026.0)$ & $-33.86(-652.3,584.61)$ & 0.912 \\
\hline \multicolumn{5}{|l|}{ TSAT, \% } \\
\hline After Course $1(\leq 1000 \mathrm{mg}, n=98 ;>1000 \mathrm{mg}, n=31)$ & $8.13(5.86,10.40)$ & $9.90(5.36,14.45)$ & $1.77(-3.33,6.87)$ & 0.492 \\
\hline Before Course $2(\leq 1000 \mathrm{mg}, n=35 ;>1000 \mathrm{mg}, \mathrm{n}=7$ ) & $0.89(-0.95,2.74)$ & $2.36(-2.21,6.94)$ & $1.47(-3.56,6.49)$ & 0.555 \\
\hline After Course $2(\leq 1000 \mathrm{mg}, n=26 ;>1000 \mathrm{mg}, n=5)$ & $14.89(8.42,21.35)$ & $13.17(-1.60,27.94)$ & $-1.72(-18.18,14.75)$ & 0.830 \\
\hline \multicolumn{5}{|l|}{ Platelets, $\times 10^{9} / \mathrm{L}$} \\
\hline After Course $1(\leq 1000 \mathrm{mg}, n=127 ;>1000 \mathrm{mg}, n=45)$ & $-17.21(-25.36,-9.06)$ & $-25.75(-39.47,-12.03)$ & $-8.54(-24.57,7.49)$ & 0.294 \\
\hline Before Course $2(\leq 1000 \mathrm{mg}, n=47 ;>1000 \mathrm{mg}, n=10)$ & $-0.91(-17.99,16.17)$ & $20.25(-18.25,58.75)$ & $21.16(-21.07,63.38)$ & 0.318 \\
\hline After Course $2(\leq 1000 \mathrm{mg}, n=49 ;>1000 \mathrm{mg}, \mathrm{n}=4)$ & $-14.74(-33.14,3.67)$ & $-24.17(-109.5,61.17)$ & $-9.44(-96.73,77.86)$ & 0.828 \\
\hline
\end{tabular}

Data presented are $\mathrm{LS}$ mean $(95 \% \mathrm{Cl})$; a negative value indicates a decrease from baseline in the relevant parameter $\mathrm{Cl}$ confidence interval, $\mathrm{Hb}$ haemoglobin, $L S$ least squares, $n$ number of patients with data, TSAT transferrin saturation 


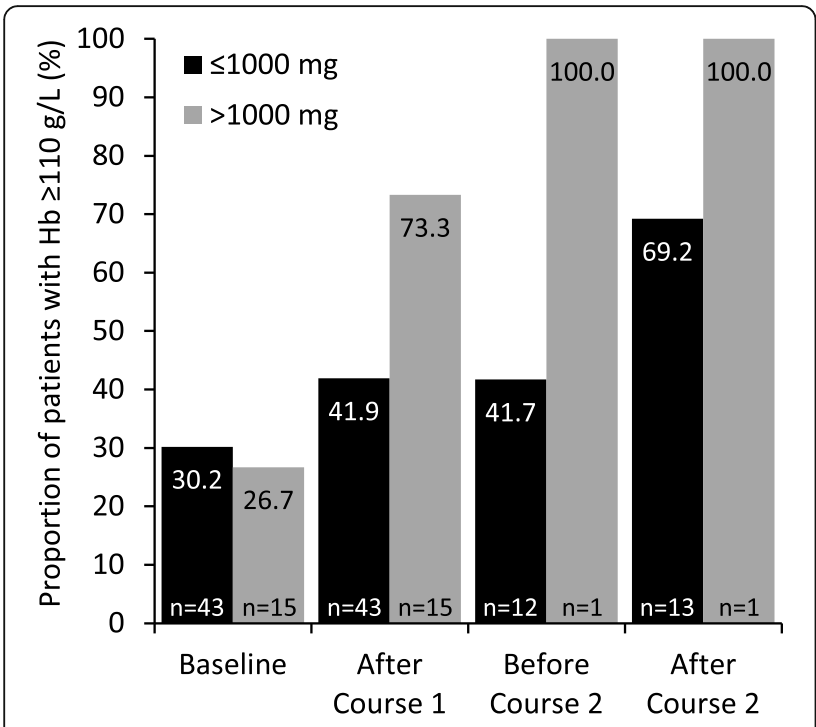

Fig. 3 Proportion of patients with $\mathrm{Hb} \geq 110 \mathrm{~g} / \mathrm{L}$ before and after IIM - patients receiving ESA at baseline.

Statistical analyses comparing higher and lower dose groups were performed for baseline and Course 1 only.

ESA erythropoiesis-stimulating agent, $\mathrm{Hb}$ haemoglobin, $n$ number of patients with data

Patients receiving $>1000 \mathrm{mg}$ IIM during Course 1 also showed a greater $\mathrm{Hb}$ response compared with the lower dose group. There was a higher proportion of patients with an $\mathrm{Hb}$ level of $\geq 110 \mathrm{~g} / \mathrm{L}$ in the $>1000 \mathrm{mg}$ dose group, irrespective of ESA treatment - this is an important observation as the UK NICE guidance recommends

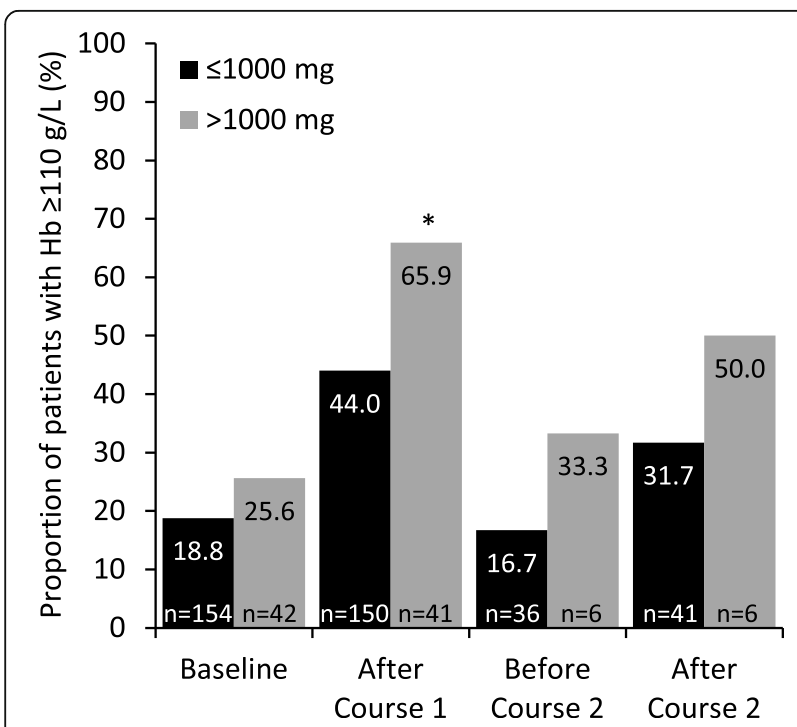

Fig. 4 Proportion of patients with $\mathrm{Hb} \geq 110 \mathrm{~g} / \mathrm{L}$ before and after IIM - patients not receiving ESA at baseline.

${ }^{*} p<0.05$ versus $\leq 1000 \mathrm{mg}$; statistical analyses comparing higher and lower dose groups were performed for baseline and Course 1 only. ESA erythropoiesis-stimulating agent, $\mathrm{Hb}$ haemoglobin, $n$ number of patients with data
Table 4 Number of appointments required, according to the dose of IIM administered in Course 1

\begin{tabular}{lll}
\hline & $\begin{array}{l}\mathbf{1 0 0 0} \mathbf{~ m g} \\
(\mathbf{N = 1 9 8})\end{array}$ & $\begin{array}{l}>1000 \mathbf{~ m g} \\
(\mathbf{N}=\mathbf{5 8})\end{array}$ \\
\hline Total number of appointments & 273 & 75 \\
Appointments per 100 patients & 137.9 & 129.3 \\
$\begin{array}{l}\text { Difference between dose groups } \\
\text { per 100 patients }\end{array}$ & & -8.6 \\
\hline
\end{tabular}

the investigation and management of anaemia in patients with an $\mathrm{Hb}$ level of $<110 \mathrm{~g} / \mathrm{L}$ [7]. Furthermore, these data support the existing evidence that $1000 \mathrm{mg}$ of IV iron may not be sufficient to achieve iron repletion in CKD patients [11]. Indeed, across all patients, the mean estimated iron need was $>1400 \mathrm{mg}$ and, in the $\leq 1000 \mathrm{mg}$ group, $82.3 \%$ of patients were 'underdosed'. These findings also highlight that the validated methods for estimating iron need are not used in all hospitals, but the clinical impact of this and the reasons why remain unclear. It is possible that underdosing occurred at some hospitals due to the perception that high doses of iron could be harmful; in addition, there is a tendency for a pragmatic attitude to dosing among clinicians, which aims to avoid iron wastage. The NIMO-CKD-UK study also reflects the fact that body weight influences the IV iron dose to some extent, as $\mathrm{Hb}$ levels and the proportion of ESA users were similar at baseline between the two dose groups. The results of this study provide support for the use of higher doses; the results show that high doses of IV iron are effective and can be administered without adverse effects.

The use of ESA requires consideration when interpreting the $\mathrm{Hb}$ response following IV iron therapy. In this study, a greater $\mathrm{Hb}$ response was observed in the higher versus the lower IV iron dose group, irrespective of baseline ESA use. However, at baseline, the monthly cumulative ESA dose was substantially higher in the lower IV iron dose group than in the higher dose group (9505 IU versus $7570 \mathrm{IU}$ ); this observation indicates that higher ESA doses were, potentially, used to compensate for the underdosing of iron, or vice versa.

Clinical guidelines recommend maintaining the $\mathrm{Hb}$ level in the range of $100-120 \mathrm{~g} / \mathrm{L}$ in CKD patients receiving ESA treatment [7]. Targeting higher $\mathrm{Hb}$ levels with ESA therapy is associated with an increased risk of stroke, hypertension, and vascular access thrombosis, as well as a possible increased risk for death, serious cardiovascular events, and end-stage renal disease [34]. The NIMO-CKD-UK study showed that the mean $\mathrm{Hb}$ levels in the $>1000 \mathrm{mg}$ IV iron group following Course 1 did not increase beyond $120 \mathrm{~g} / \mathrm{L}$, even in patients receiving ESA at baseline, offering reassurance that higher initial doses of IV iron do not elevate $\mathrm{Hb}$ to levels that are 
associated with safety risks (an $\mathrm{Hb}$ level $>120 \mathrm{~g} / \mathrm{L}$ is acceptable for patients not receiving ESA [35]). Indeed, the results of the PIVOTAL study support this theory [12]. More specifically, it has been postulated that ESAinduced iron deficiency leads to an increase in platelet count that can cause thrombocytosis - a risk factor for thrombovascular events [36]. Evidence suggests that IV iron can reduce the platelet count [37], thereby lowering the risk of thrombocytosis [38]. The NIMO-CKD-UK study supports this theory by showing a reduction in platelet count following IV iron treatment, in both dose groups (Table 3). Platelet counts were higher in the lower dose group, possibly a reflection that these patients did not receive their full iron need and retained a certain degree of iron deficiency (known to increase platelet count [36]). The cumulative dose of ESA at baseline was also higher in the lower IV iron dose group.

The observed improvements in FACIT-Fatigue score confirm the established beneficial effect of IV iron on quality of life [39]. A lack of a statistically significant difference between the higher and lower dose groups cannot be explained easily. It may be that the scale is not sensitive enough to capture a difference between the groups, or the improvements in $\mathrm{Hb}$ between the two groups may not have been large enough to translate into a differential benefit on quality of life. In addition, it cannot be ruled out that a lack of a detectable difference between the two groups was due to the small patient numbers.

The safety data are consistent with the established profile for IIM showing a low risk of ADRs in patients with CKD from clinical trials and other observational studies $[8,17,18,20-22]$. For example, the FERWONNEPHRO study - an RCT of over 1000 IIM-treated patients - reported that IIM was well tolerated with a low ADR rate [19]. In addition, the FERWON-NEPHRO study showed a significantly lower rate of cardiovascular events with IIM versus the comparator, iron sucrose [19]. The proportion of deaths that occurred during the NIMO-CKD-UK study is similar to that reported in a previous large observational study [20], and should be considered within the context of a real-world patient population with chronic illness and multiple morbidities. The time interval between the last IV iron administration and death supports the investigators' judgement that these events are unrelated to IV iron treatment.

Considering the study limitations, the pragmatic design dictated that no instructions were provided to the investigators on dosing, time to the assessment of treatment response, or how to assess the need for retreatment and, therefore, no information on these practices was collected. Indeed, the baseline data indicate that the tests used to identify iron deficiency (ferritin and/or TSAT) may have varied between centres. However, the aim was to observe treatment routines in clinical practice to provide guidance for optimised strategies for IV iron treatment. In addition, the small number of patients who received a second course of IV iron treatment hinders interpretation of the data collected after repeated dosing, and prevents any conclusions in relation to a dose effect. Although the study did not collect data for ESA therapy post-baseline to determine whether IV iron therapy reduced the need for ESAs, there is no reason to expect that substantial changes in the ESA regimen would have been necessary. It is, therefore, assumed that higher ESA doses were used in the $\leq 1000 \mathrm{mg}$ group throughout the study course. Finally, this study did not evaluate the longer-term implications of the different doses of IV iron; future studies are needed to examine any such impact by analysing, for example, number of hospitalisations.

\section{Conclusions}

The NIMO-CKD-UK study shows that current clinical practice with IIM is effective in ND-CKD patients. In a real-world setting in the UK, a higher initial dose $(>1000 \mathrm{mg})$ of IIM reduced the probability of retreatment, achieved a greater $\mathrm{Hb}$ response irrespective of ESA treatment, and reduced the total number of appointments required compared with a lower dose $(\leq 1000 \mathrm{mg})$. Use of a higher initial dose treatment approach could be considered to reduce hospital visits during the COVID-19 pandemic.

\section{Supplementary Information}

Supplementary information accompanies this paper at https://doi.org/10. 1186/s12882-020-02180-2.

Additional file 1: Table S1. Additional blood and iron parameters at baseline. Table S2. Dose of IIM administered during Course 1, according to ESA treatment. Table S3. Probability of retreatment with IV iron, according to the IIM dose administered during Course 1. Table S4. Mean change in $\mathrm{Hb}$ from baseline, according to ESA treatment. Table S5. Mean change in FACIT-Fatigue Total score from baseline to after Course 1.

\begin{abstract}
Abbreviations
ADR: adverse drug reaction; ANCOVA: analysis of covariance; Cl: confidence interval; CKD: chronic kidney disease; eGFR: estimated glomerular filtration rate; ESA: erythropoiesis-stimulating agent; FACIT: Functional Assessment of Chronic Illness Therapy; Hb: haemoglobin; IDA: iron deficiency anaemia; IIM: iron isomaltoside; IU: international unit; IV: intravenous; KDIGO: Kidney Disease Improving Global Outcomes; LS: least squares; n: number of patients with data; ND-CKD: non-dialysis chronic kidney disease; NICE: National Institute for Health and Care Excellence; NIMO: Non-Interventional Monofer; $\mathrm{RCT}$ : randomised controlled trial; SD: standard deviation; TSAT: transferrin saturation
\end{abstract}

Acknowledgements

The authors thank all investigators and study staff for their contribution to the study, and especially Dr. T Doulton and Dr. J Neary. Writing support was provided by Cambridge Medical Communication Ltd. (Cambridge, UK). 


\section{Authors' contributions}

PAK, SB, MS, RD, SL, AM, DR, NP, KM, and JM were involved with the concept and design of the study, the acquisition, analysis, and interpretation of data, the drafting of the paper and revising it critically for intellectual content. All authors (PAK, SB, MS, RD, SL, AM, DR, NP, KM, and JM) also approved the final manuscript and agree to be accountable for all aspects of the work.

\section{Funding}

Pharmacosmos UK Ltd. was involved in designing this study, and it acted as the study sponsor. Pharmacosmos UK Ltd. provided research funding to the participating institutions, and financial support that allowed analysis and interpretation of the data and the preparation of the manuscript.

\section{Availability of data and materials}

The datasets used and/or analysed during the current study are available from the corresponding author on reasonable request.

\section{Ethics approval and consent to participate}

The Health Research Authority and the Regional Ethics Committee in the UK (reference: 15/EM/0303) approved the study and all subsequent protocol amendments. All study participants gave written informed consent before inclusion into the study, and the study was performed in accordance with Good Pharmacovigilance Practices and the European Medicines Agency criteria for non-interventional studies [40].

\section{Consent for publication}

Not applicable.

\section{Competing interests}

Pharmacosmos UK Ltd. sponsored the study. PAK and SB have received consultancy fees, speaker honoraria and educational grants from Pharmacosmos and Vifor Pharma. MS is an employee of Pharmacosmos UK Ltd. RD has received consultancy fees, speaker honoraria and educational grants from Pharmacosmos. DR has received consultancy fees and educational grants from Pharmacosmos. SL has received consultancy fees from Pharmacosmos. AM and KM have received consultancy fees from Pharmacosmos and Vifor Pharma. NP has received speaker honoraria from Pharmacosmos. JM has no conflicts of interest.

\section{Author details}

'Salford Royal NHS Foundation Trust, Salford, UK. ${ }^{2}$ Hull University Teaching Hospitals NHS Trust, Hull, UK. ${ }^{3}$ Pharmacosmos UK, Reading, UK. ${ }^{4}$ Sunderland Royal Hospital, Sunderland, UK. ${ }^{5}$ Royal Sussex County Hospital, Brighton, UK. ${ }^{6}$ Morriston Hospital, Swansea, UK. ${ }^{7}$ The James Cook University Hospital, Middlesbrough, UK. ${ }^{8}$ Addenbrooke's University Hospital, Cambridge, UK. ${ }^{9}$ Royal London University Hospital, London, UK. ${ }^{10}$ Royal Devon and Exeter University Hospital, Exeter, UK.

\section{Received: 12 March 2020 Accepted: 19 November 2020}

\section{Published online: 10 December 2020}

\section{References}

1. Gotloib L, Silverberg D, Fudin R, Shostak A. Iron deficiency is a common cause of anemia in chronic kidney disease and can often be corrected with intravenous iron. J Nephrol. 2006:19(2):161-7.

2. McClellan W, Aronoff SL, Bolton WK, Hood S, Lorber DL, Tang KL, et al. The prevalence of anemia in patients with chronic kidney disease. Curr Med Res Opin. 2004;20(9):1501-10

3. Stauffer ME, Fan T. Prevalence of anemia in chronic kidney disease in the United States. PLoS One. 2014;9(1):e84943.

4. Eriksson D, Goldsmith D, Teitsson S, Jackson J, van Nooten F. Cross-sectional survey in CKD patients across Europe describing the association between quality of life and anaemia. BMC Nephrol. 2016;17(1):97.

5. Kovesdy CP, Trivedi BK, Kalantar-Zadeh K, Anderson JE. Association of anemia with outcomes in men with moderate and severe chronic kidney disease. Kidney Int. 2006:69(3):560-4.

6. Dowling TC. Prevalence, etiology, and consequences of anemia and clinical and economic benefits of anemia correction in patients with chronic kidney disease: an overview. Am J Health Syst Pharm. 2007;64(13 Suppl 8):S3-7.
7. National Institute for Health and Care Excellence (NICE). Chronic kidney disease: managing anaemia. NICE guideline (NG8). June 2015. www.nice.org uk/guidance/ng8. Accessed 19 Aug 2019

8. Kalra PA, Bhandari S, Saxena S, Agarwal D, Wirtz G, Kletzmayr J, et al. A randomized trial of iron isomaltoside 1000 versus oral iron in non-dialysisdependent chronic kidney disease patients with anaemia. Nephrol Dial Transplant. 2016;31(4):646-55.

9. Shepshelovich D, Rozen-Zvi B, Avni T, Gafter U, Gafter-Gvili A. Intravenous versus oral iron supplementation for the treatment of anemia in CKD: an updated systematic review and meta-analysis. Am J Kidney Dis. 2016;68(5): $677-90$

10. Evstatiev R, Marteau P, Iqbal T, Khalif IL, Stein J, Bokemeyer B, et al. FERGI study group. FERGIcor, a randomized controlled trial on ferric carboxymaltose for iron deficiency anemia in inflammatory bowel disease. Gastroenterology. 2011;141(3):846-53.

11. Koch TA, Myers J, Goodnough LT. Intravenous iron therapy in patients with iron deficiency anemia: dosing considerations. Anemia. 2015;2015:763576

12. Macdougall IC, White C, Anker SD, Bhandari S, Farrington K, Kalra PA, et al. PIVOTAL investigators and committees. Intravenous iron in patients undergoing maintenance hemodialysis. N Engl J Med. 2019;380(5):447-58.

13. Onken JE, Bregman DB, Harrington RA, Morris D, Buerkert J, Hamerski D, et al. Ferric carboxymaltose in patients with iron-deficiency anemia and impaired renal function: the REPAIR-IDA trial. Nephrol Dial Transplant. 2014; 29(4):833-42.

14. Macdougall IC, Bock AH, Carrera F, Eckardt KU, Gaillard C, Van Wyck D, et al. FIND-CKD study investigators. FIND-CKD: a randomized trial of intravenous ferric carboxymaltose versus oral iron in patients with chronic kidney disease and iron deficiency anaemia. Nephrol Dial Transplant. 2014;29(11): 2075-84.

15. Monofer $^{\circledast}$ (iron isomaltoside 1000). Summary of product characteristics. (c) Pharmacosmos UK Limited. May 2020

16. Kalra PA, Bhandari S. Efficacy and safety of iron isomaltoside (Monofer $\left.{ }^{\circledR}\right)$ in the management of patients with iron deficiency anemia. Int J Nephrol Renovasc Dis. 2016:9:53-64.

17. Wikström B, Bhandari S, Barany P, Kalra PA, Ladefoged S, Wilske J, Thomsen LL. Iron isomaltoside 1000: a new intravenous iron for treating iron deficiency in chronic kidney disease. J Nephrol. 2011;24(5):589-96.

18. Bhandari S, Kalra PA, Kothari J, Kalra PA, Ladefoged S, Wilske J, Thomsen LL. A randomized, open-label trial of iron isomaltoside 1000 (Monofer ${ }^{\circledR}$ ) compared with iron sucrose $\left(V_{e n o f e r}{ }^{\circledR}\right)$ as maintenance therapy in haemodialysis patients. Nephrol Dial Transplant. 2015;30(9):1577-89.

19. Bhandari A, Kalra PA, Berkowitz M, Belo D, Thomsen LL, Wolf M. A randomized, open-label, comparative trial of iron isomaltoside 1000 and iron sucrose in chronic kidney disease patients (FERWON-NEPHRO). Nephrol Dial Transplant. 2020; https://doi.org/10.1093/ndt/gfaa011.

20. Biggar $P$, Leistikow $F$, Walper A. A prospective observational study of effectiveness and safety of iron isomaltoside in patients with chronic renal failure and iron deficiency anemia. Clin Nephrol. 2016;86(12):310-8.

21. Jensen G, Gøransson LG, Fernström A, Furuland H, Christensen JH. Treatment of iron deficiency in patients with chronic kidney disease: a prospective observational study of iron isomaltoside (NIMO Scandinavia). Clin Nephrol. 2019;91(4):246-53.

22. Sivakumar C, Jubb VM, Lamplugh A, Bhandari S. Safety of intravenous iron Cosmofer and Monofer therapy in peritoneal dialysis and non-dialysisdependent chronic kidney disease patients. Perit Dial Int. 2019:39(2):192-5.

23. Kidney Disease: Improving Global Outcomes (KDIGO). Clinical practice guideline for anemia in chronic kidney disease. Kidney Int Suppl. 2012;2:279-335.

24. Ganzoni AM. Intravenous iron-dextran: therapeutic and experimental possibilities. Schweiz Med Wochenschr. 1970;100(7):301-3.

25. Acaster S, Dickerhoof R, DeBusk K, Bernard K, Strauss W, Allen LF. Qualitative and quantitative validation of the FACIT-fatigue scale in iron deficiency anemia. Health Qual Life Outcomes. 2015;13(1):60

26. Aranesp ${ }^{\oplus}$ (darbepoetin alfa). Summary of product characteristics. (c) Amgen Limited. November 2019.

27. Frigstad SO, Haaber A, Bajor A, Fallingborg J, Hammarlund P, Bonderup OK, et al. The NIMO Scandinavian study: a prospective observational study of iron isomaltoside treatment in patients with iron deficiency. Gastroenterol Res Pract. 2017:2017:4585164

28. Pollock RF, Muduma G. A budget impact analysis of parenteral iron treatments for iron deficiency anemia in the UK: reduced resource utilization with iron isomaltoside 1000. Clinicoecon Outcomes Res. 2017;9:475-83. 
29. Pollock RF, Muduma G. Intravenous iron treatments for iron deficiency anemia in inflammatory bowel disease: a budget impact analysis of iron isomaltoside 1000 (Monofer) in the UK. Expert Opin Drug Deliv. 2017;14(12): 1439-46.

30. Pollock RF, Muduma G. An economic evaluation of iron isomaltoside 1000 versus ferric carboxymaltose in patients with inflammatory bowel disease and iron deficiency anemia in Denmark. Adv Ther. 2018;35(12):2128-37.

31. Bhandari S. Update of a comparative analysis of cost minimization following the introduction of newly available intravenous iron therapies in hospital practice. Ther Clin Risk Manag. 2011;7:501-9.

32. National Health Service (NHS). Clinical guide for the management of remote consultations and remote working in secondary care during the coronavirus pandemic. Version 1. 27 March 2020.

33. Centers for Disease Control and Prevention (CDC) website. Healthcare facilities: managing operations during the COVID-19 pandemic. https:// www.cdc.gov/coronavirus/2019-ncov/hcp/guidance-hcf.html. Accessed 9 Nov 2020.

34. Palmer SC, Navaneethan SD, Craig JC, Johnson DW, Tonelli M, Garg AX, et al. Meta-analysis: erythropoiesis-stimulating agents in patients with chronic kidney disease. Ann Intern Med. 2010;153(1):23-33.

35. Mikhail A, Brown C, Williams JA, Mathrani V, Shrivastava R, Evans J, et al. Renal association clinical practice guideline on anaemia of chronic kidney disease. BMC Nephrol. 2017;18:345.

36. Dahl NV, Henry DH, Coyne DW. Thrombosis with erythropoietic stimulating agents - does iron-deficient erythropoiesis play a role? Semin Dial. 2008; 21(3):210-1.

37. Hazara AM, Bhandari S. Intravenous iron administration is associated with reduced platelet counts in patients with chronic kidney disease. J Clin Pharm Ther. 2015;40(1):20-3.

38. Henry DH, Dahl NV, Auerbach MA. Thrombocytosis and venous thromboembolism in cancer patients with chemotherapy induced anemia may be related to ESA induced iron restricted erythropoiesis and reversed by administration of IV iron. Am J Haematol. 2012;87(3):308-10.

39. Strauss $W E$, Auerbach $M$. Health-related quality of life in patients with iron deficiency anemia: impact of treatment with intravenous iron. Patient Relat Outcome Meas. 2018;9:285-98.

40. European Medicines Agency. Directive 2001/20/EC of the European Parliament and of the Council Article 2(c). 2001.

\section{Publisher's Note}

Springer Nature remains neutral with regard to jurisdictional claims in published maps and institutional affiliations.

\section{Ready to submit your research? Choose BMC and benefit from}

- fast, convenient online submission

- thorough peer review by experienced researchers in your field

- rapid publication on acceptance

- support for research data, including large and complex data types

- gold Open Access which fosters wider collaboration and increased citations

- maximum visibility for your research: over $100 \mathrm{M}$ website views per year

At $\mathrm{BMC}$, research is always in progress.

Learn more biomedcentral.com/submissions 